\title{
2017:9(3):147-153. DOI: 10.18869/acadpub.ijwjph.9.3.147 \\ Relationship between Sport Participation and Social Capital in Veterans and Disabled People; Case Study of Kerman City, Iran
}

\section{ART ICLE INF O}

\section{Article Type}

Descriptive Study

\section{Authors}

Miryousefi S.J.* $P h D$,

Rahimi Y. ${ }^{1} M S c$

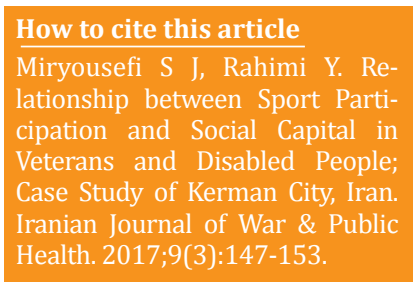

*Physical Education Department, Literature \& Humanities Faculty, University of Vali-e-Asr, Rafsanjan, Iran

${ }^{1}$ Education District No. One, Kerman, Iran

\section{Correspondence}

Address: Literature Faculty, University of Vali-e-Asr, Imam Khomeini Square, Rafsanjan, Iran. Post Box: 518

Phone: +98 (34) 31312068

Fax: +98 (34) 31312068

s.j.miryousefi@gmail.com

\section{Article History}

Received: February 4, 2017

Accepted: April 17, 2017

ePublished: July 27, 2017

\section{A B S T R A C T}

Aims Acquiring social capital through participation in sports activities is very important for all segments of society, especially the veterans and disabled people. The purpose of this study was to investigate the relationship between sports participation and social capital of veterans and disabled people.

Instrument \& Methods In this descriptive-correlational study in 2015, A total of 170 war veterans and disabled male and female sportsmen of Kerman (92 veterans and 78 disabled persons) were studied using the available sampling method. The used instrument was a researcher-made questionnaire on sports participation and social capital of veterans and disabled, which validity and reliability were confirmed. Data were analyzed by SPSS 19 software using Pearson correlation coefficient test.

Findings There was a positive significant correlation between sport participation and social participation ( $\mathrm{r}=0.292)$ and also between sports participation and social capital $(\mathrm{r}=0.176)$ in veterans and disabled people $(\mathrm{p}<0.05)$, but there was not a significant correlation between sport partnership and social support and social security in veterans and disabled $(p>0.05)$. Conclusion The sports participation of veterans and disabled people in Kerman has a direct and positive correlation with their social capital.

Keywords Sports; Social Capital; Veterans; Disabled Persons

\section{I T A T I O N L I N K S}

[1] How to spend the leisure time of female students of Islamic Azad universities ... [2] Relationship between social capital and participation in ... [3] A survey about impact of social support on the ... [4] Sport and ... [5] The relationship between family social capital and sport ... [6] Development through sport: Building social capital ... [7] Bowling alone: The collapse and revival ... [8] Social capital in the creation of human ... [9] Position on how to spend leisure time sports activities for the Veterans ... [10] Disability in Iran: Prevalence, characteristics, and ... [11] A study of chaharbagh avenue as a case study ... [12] Study and prioritizing the factors and barriers ... [13] The relationship between exercise and mental health ... [14] The participation motive in the ... [15] What happens to people before and after ... [16] The examination of the relationship between ... [17] The role of physical activity and sport in ... [18] How to spend the leisure time of veterans and ... [19] Physical activity and quelity ... [20] A study of the barriers to participation [21] The role of sport in leisure time spend of students in Islamic Azad University ... [22] Barrierstoandfacilitatorsofsportsparticipationforpeoplewith physicaldisabilities... [23] The relationship between participation motives and sport commitment [24] Relationship between sport participation motivation with ... [25] Sport commitment in wheelchair basketball: An interpretive look into the lives of ... [26] Compare body image of disabled athletes with disabilities ... [27] Sport in society- issues and ... [28] A Survey about the relationship between social capital ... [29] Social capital and leisure time physical activity: A population based multilevel analysis in ... [30] Leisure time physical activity and ... [31] AIndividual, social and environmental correlates of physical activity ... [32] Sport and social ... [33] Community social ... [34] Physical activity patterns of Singaporeans ... [35] Social capital and change in psychological ... [36] The study of the relationship between social trust and feeling of social security among ... [37] Motivational Factor Analysis of Student Participation in University's ... [38] Sport as a vehicle for socialization and maintenance ... [39] A study on the difference of social capital ... [40] Investigate the relationship between social capital and sport ... 
است كه طى آن فعاليتها و اعمال افراد از يكديخر تاثيريذيرى

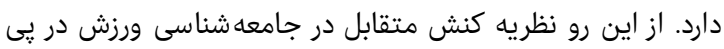

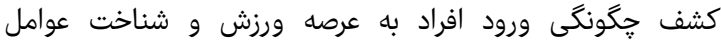

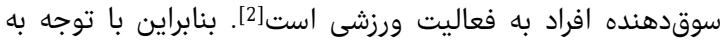

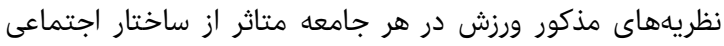

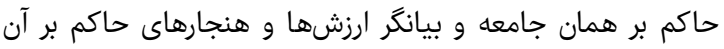

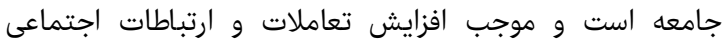

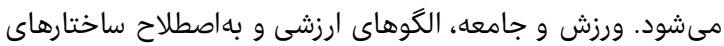

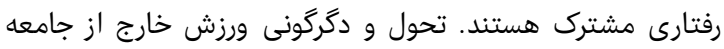

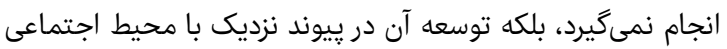

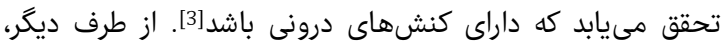

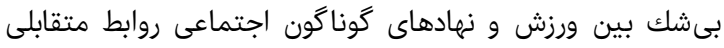

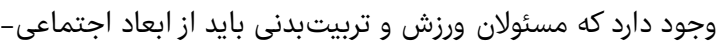

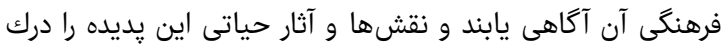

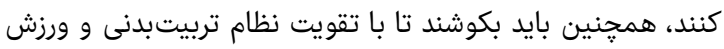

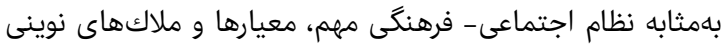

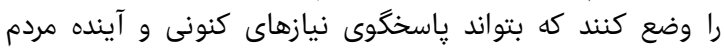

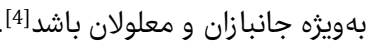

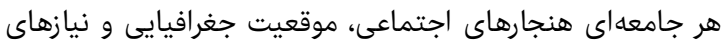

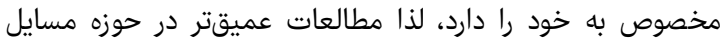

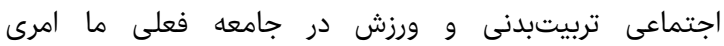

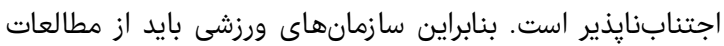

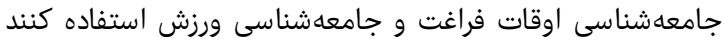

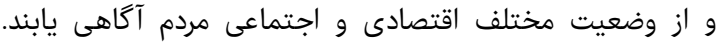

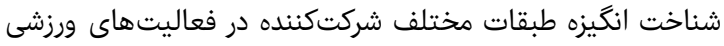

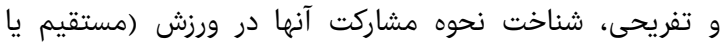

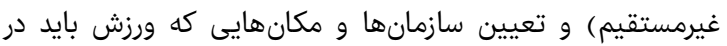

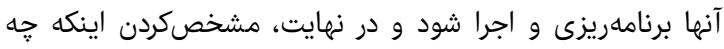

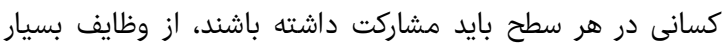

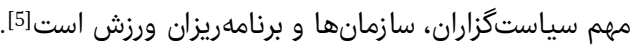

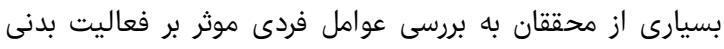

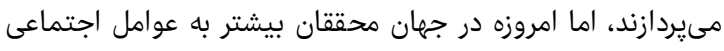

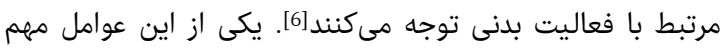

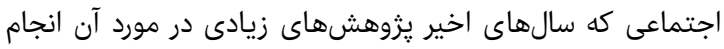

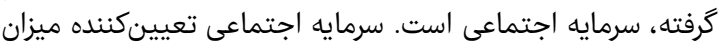

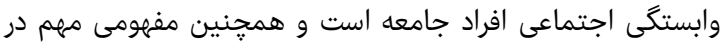

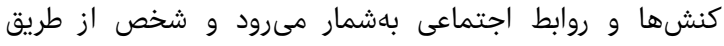

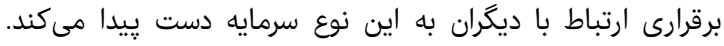

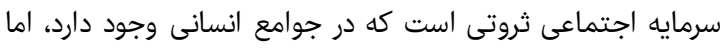

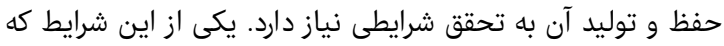

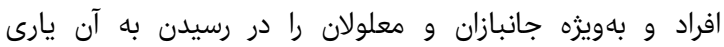

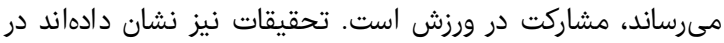

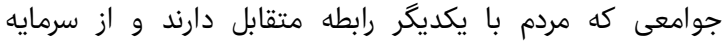

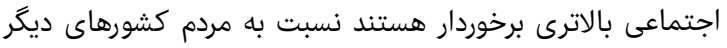

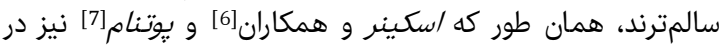

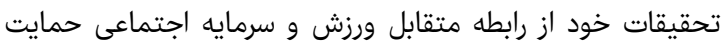

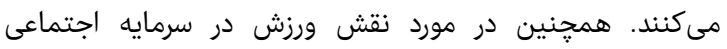

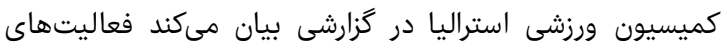

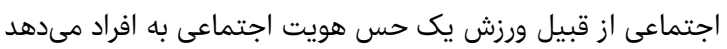

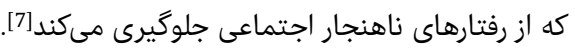

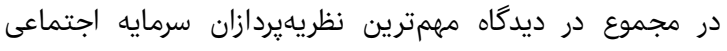

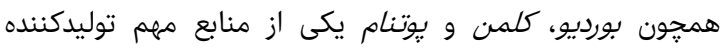

دوره 9، شماره ץ، تابستان عوسا

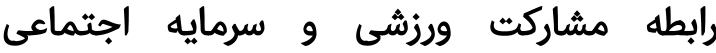 جانبازان و معلولان؛ مطالعه موردى شهر كرمان}

\author{
سيدجليل ميريوسفى " PhD

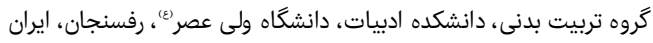 \\ يداله رحيمى MSc
} آموزش و يرورش ناحيه يك،كرمان، ايران קكيده

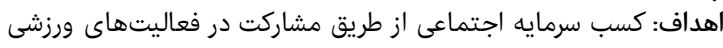

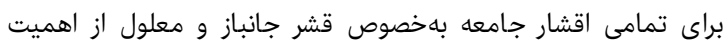

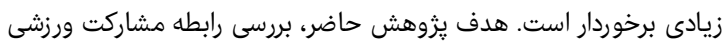

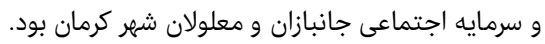

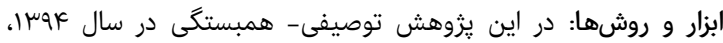

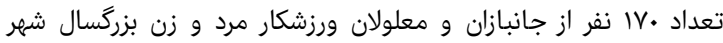

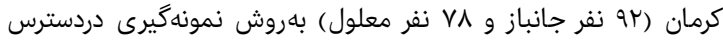

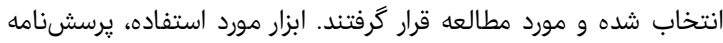

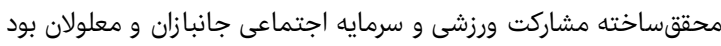

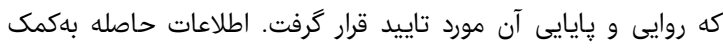

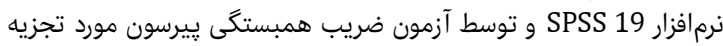

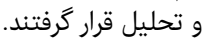

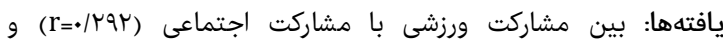

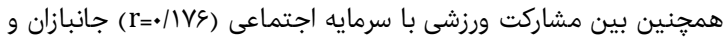

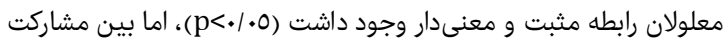

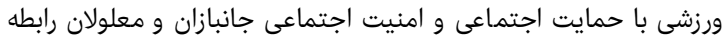

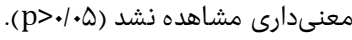

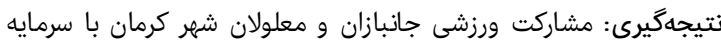

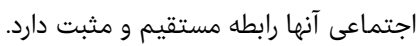

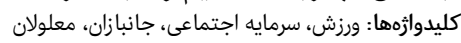

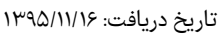

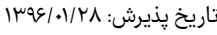

s.j.miryousefi@gmail.com : تاريخ بذيرش:

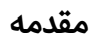

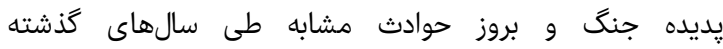
خسارتهاى مادى، انسانى و اجتماعى زيادى روى را بلهدنبال داشته

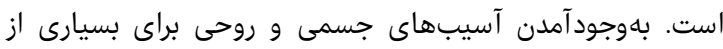

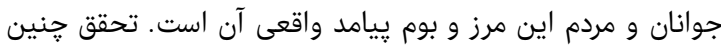

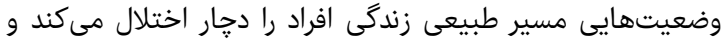

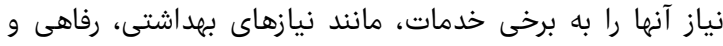

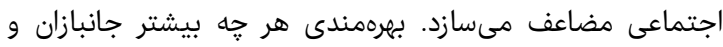

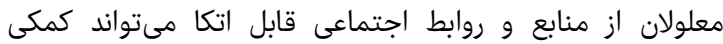

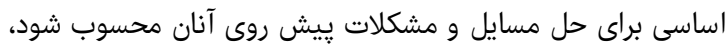

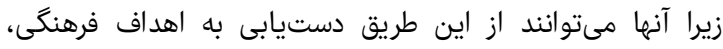

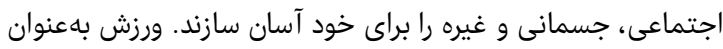

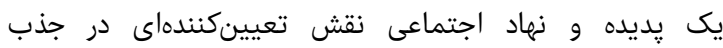
ارزشهاى فرهنگى و حفظ سلامت جسمى و روحى ارئى انسانها

دارد[1].

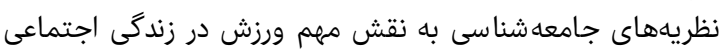

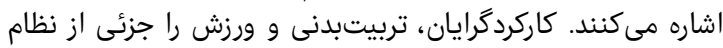

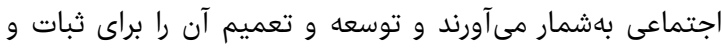

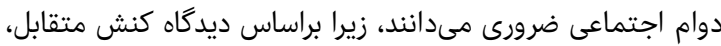
زندگى اجتماعى مستلزم نوعى ارتباط است. كنش مترد متقابل فرآيندى 


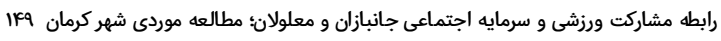

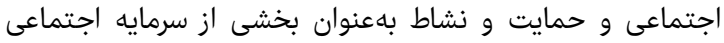

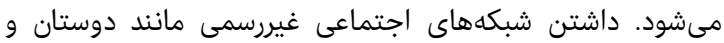

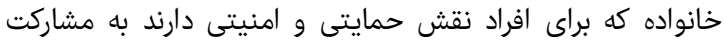

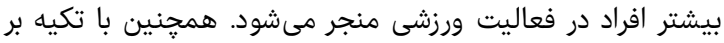

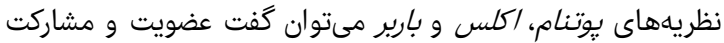

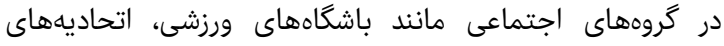

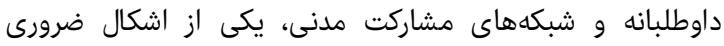

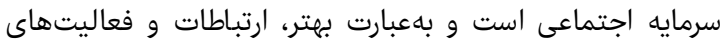

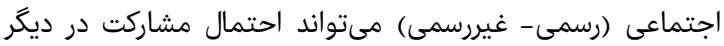

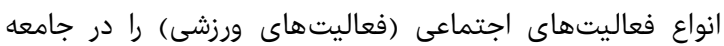

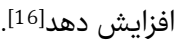

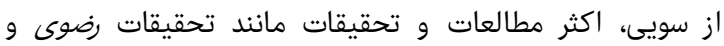

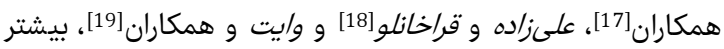

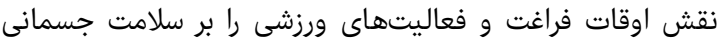

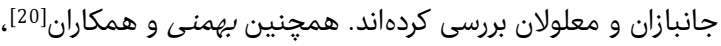

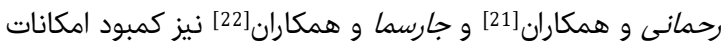

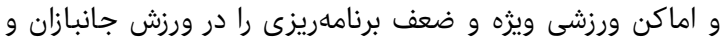

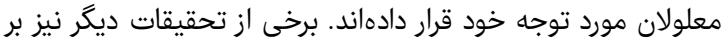

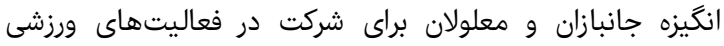
تاكيد دارند كه مىتوان از تحقيقان معلون على بحسينى و همكاران [23]،

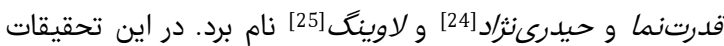

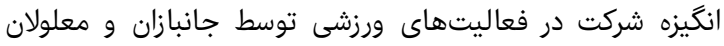

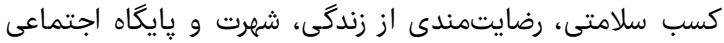

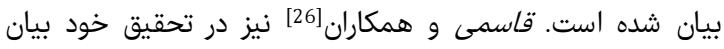

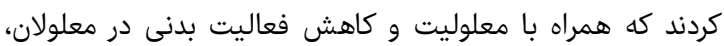

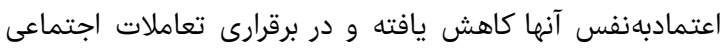

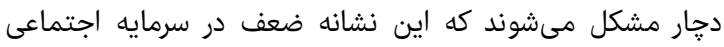
معلولان است.

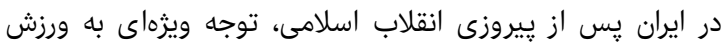

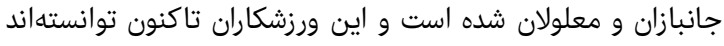

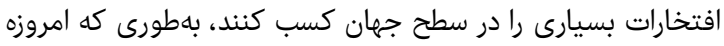

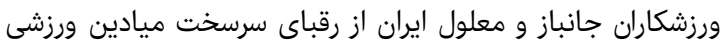

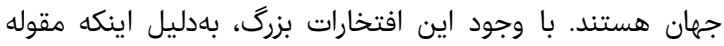

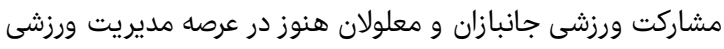

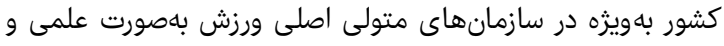

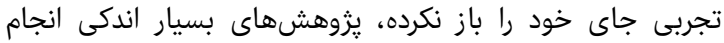

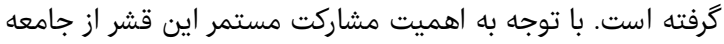

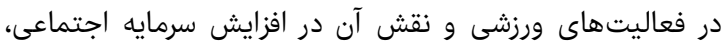

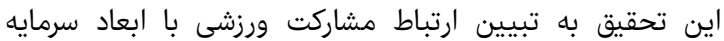

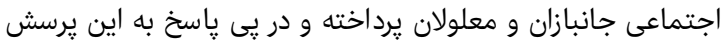

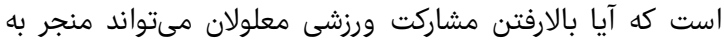

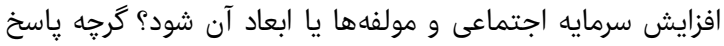

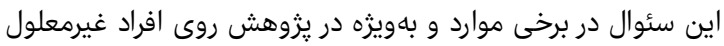

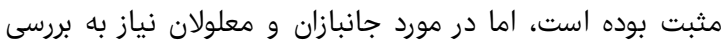
داشت.

بنابراين هدف يثوهش حاضر، بررسى رابطه مشاركت ورزشى و

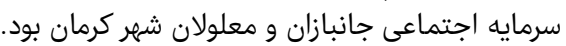

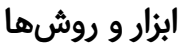

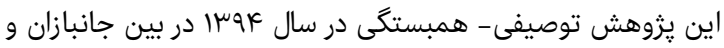

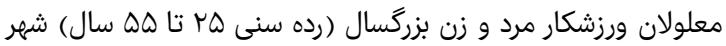

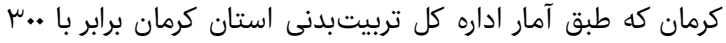

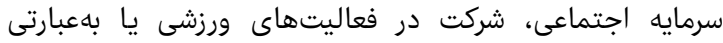

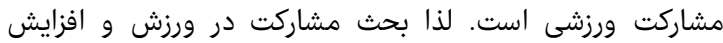

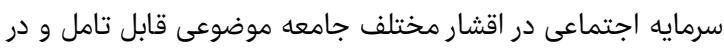

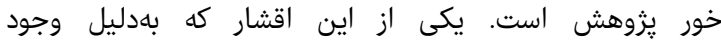

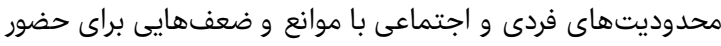

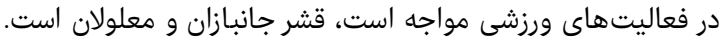

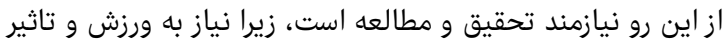

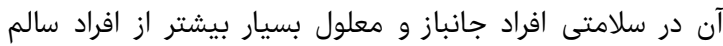

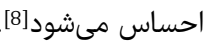

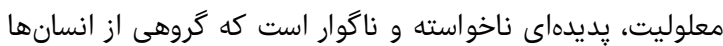

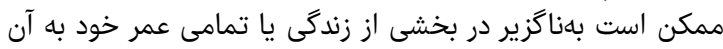

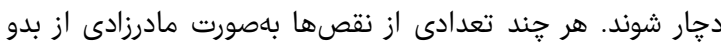

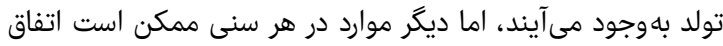

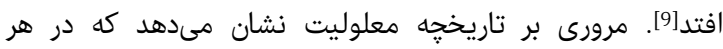

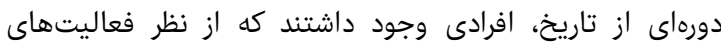

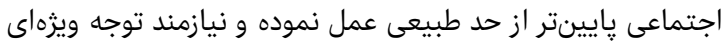

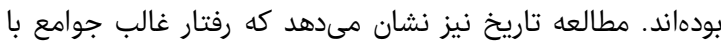

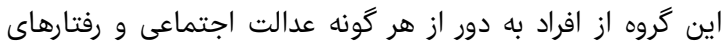

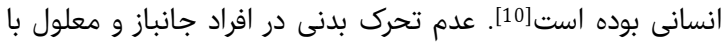

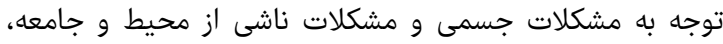

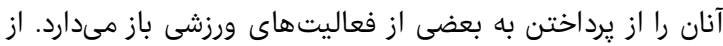

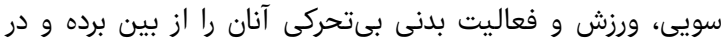

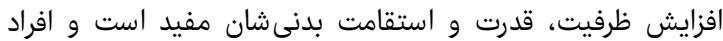

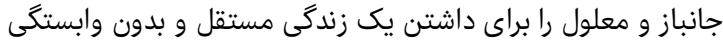

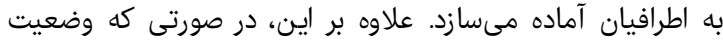

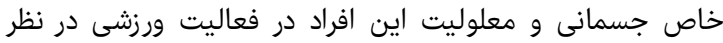

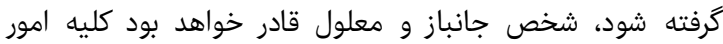

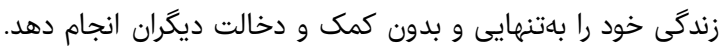

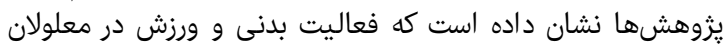

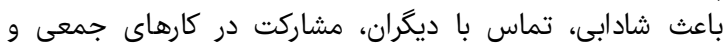

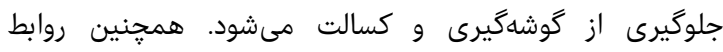

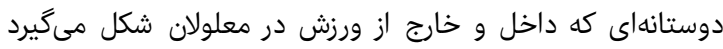

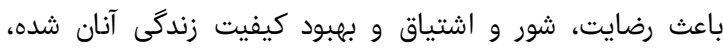

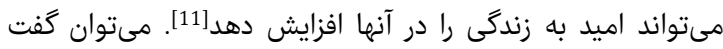

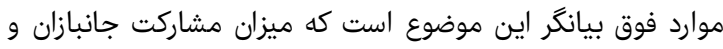

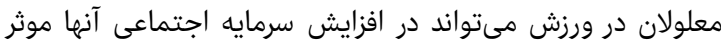

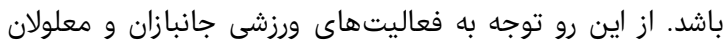

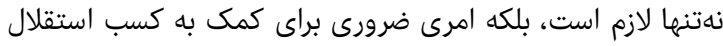

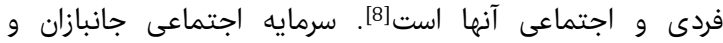

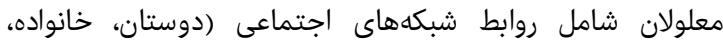

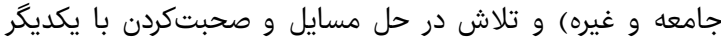

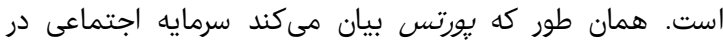

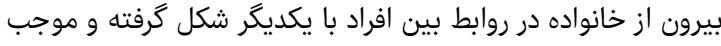

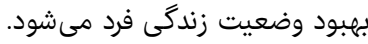

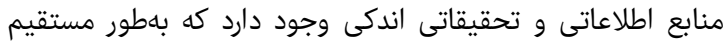

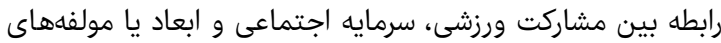

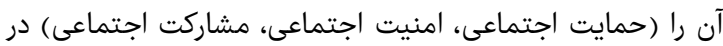

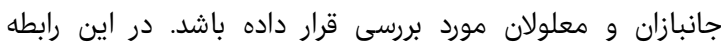

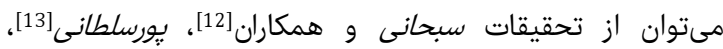

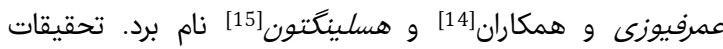

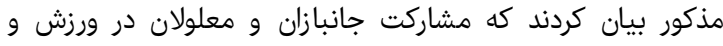

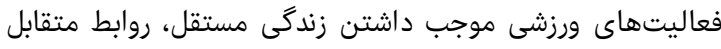


با حمايت اجتماعى و امنيت اجتماعى جانبازان و معلولان رابطه

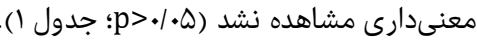

جدول () ميانكين آمارى نمرات سرمايه اجتماعى و مولفههاى آن و ضرايب

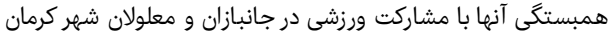

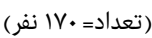

\begin{tabular}{|c|c|c|c|}
\hline معنى سطارى & ضا مشاركت همبستى ورزشى & ميانكين & متغيرها \\
\hline.$/ k \mu \mu$ &.$/ 11 \mu$ & $11 / \varepsilon \varepsilon \pm r / \varepsilon \mu$ & امنيت اجتماعى \\
\hline.$/ q \mu F$ & .1 .09 & $\mu / / 1 . \pm q / \mu$. & حمايت اجتماعى \\
\hline $.1 . .1$ & . I Tar & WI/OIEQ/qT & مشاركت اجتماعى \\
\hline. $.4 r$ & •/IVS & 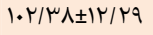 & سرمايه اجتماعى \\
\hline
\end{tabular}

بحث

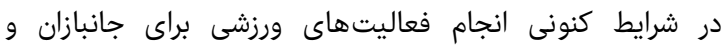

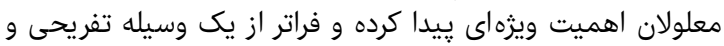

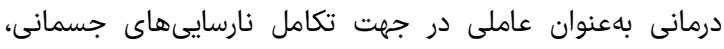

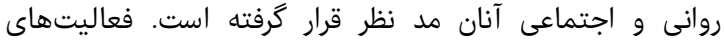

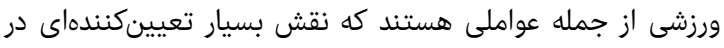

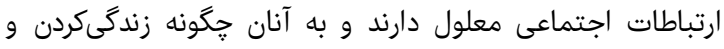

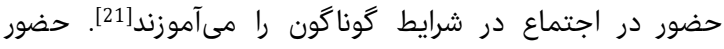

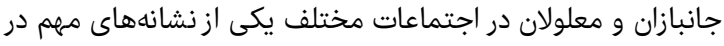

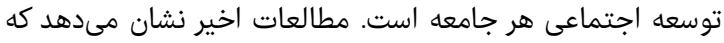

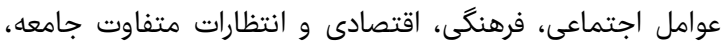

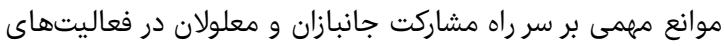

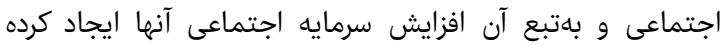

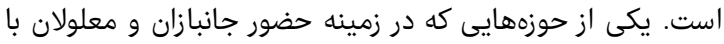

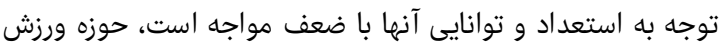

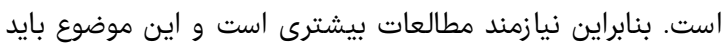

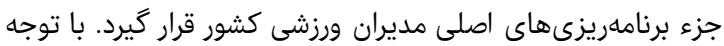

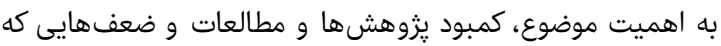

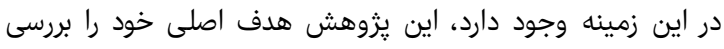
رابطه بين مشاركت ورزشى و سرمايه اجتماعى جانبازان و معلولانان

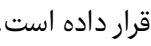

نتايج نشان داد بين مشاركت ورزشى و مشاركت اجتماعى جانبازان

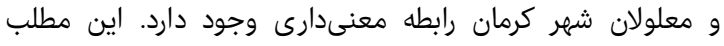

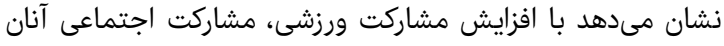

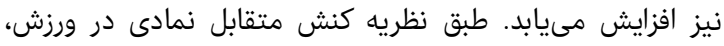

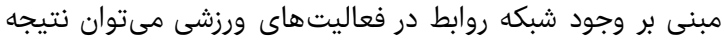

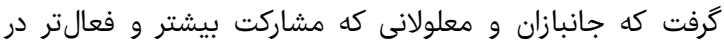

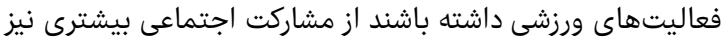

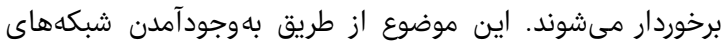

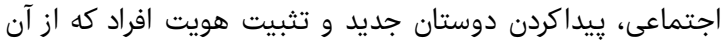

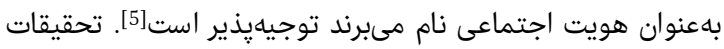

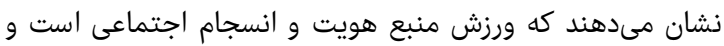

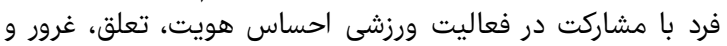

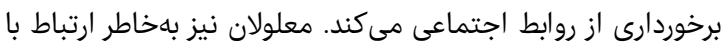

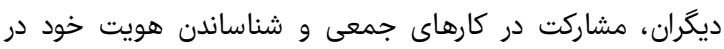

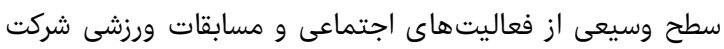

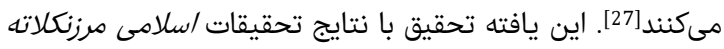

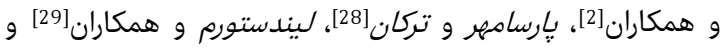

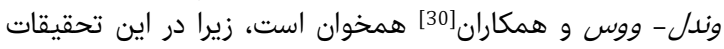
اشاره شده است كه كاهش ميزان فعاليت بدنى در اوقات فراغت دران به به

دوره 9، شماره س، تابستان عوس1

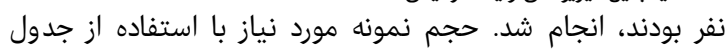

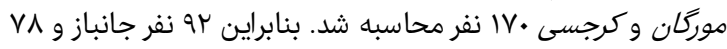

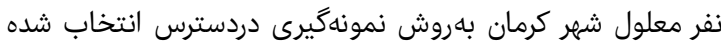
و مورد مطالعه قرار كرفتند.

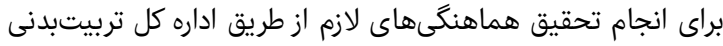

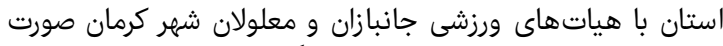

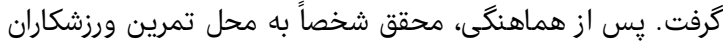

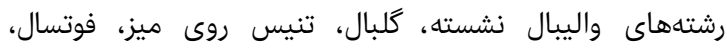

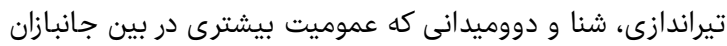

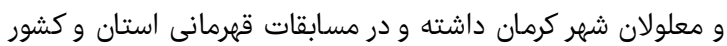

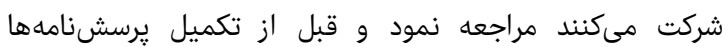

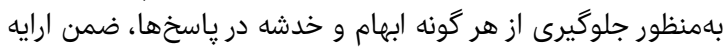

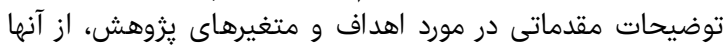

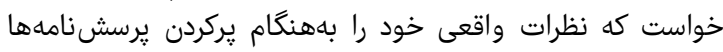

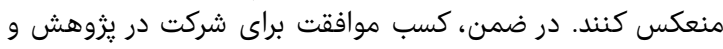

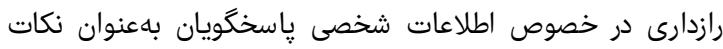

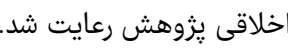

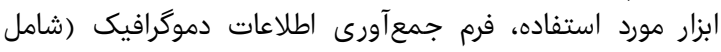

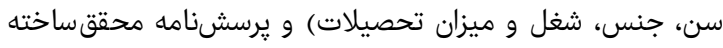

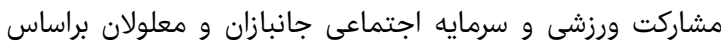

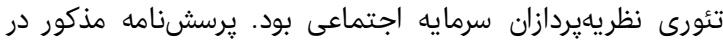

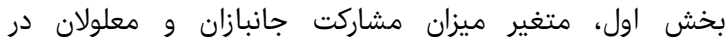

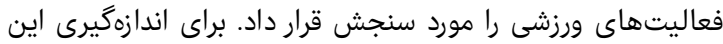

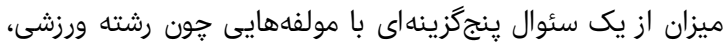

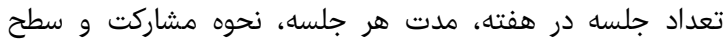

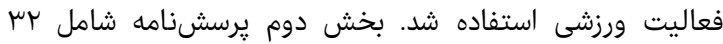

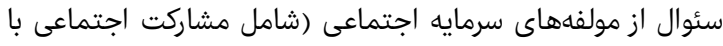

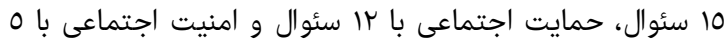

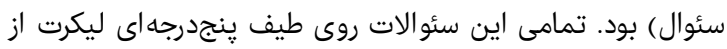

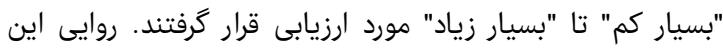

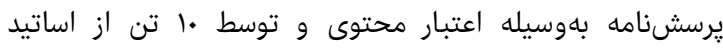

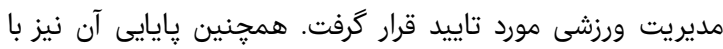

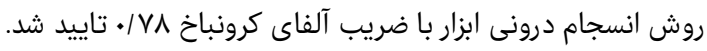

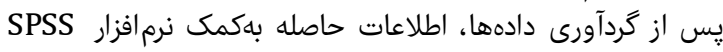

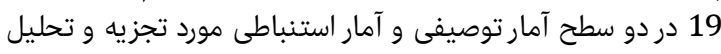

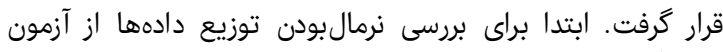

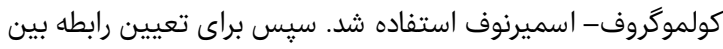

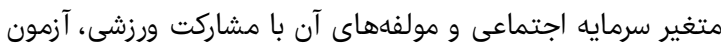
ضر يب همبستگى يِيرسون مورد استفاده قرار كرفت.

\section{يافتهها}

ميانكين سنى نمونها

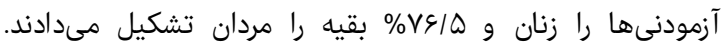
\%VN/A

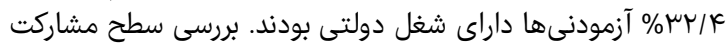

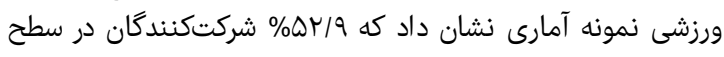

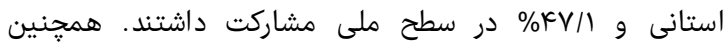

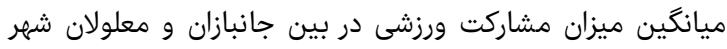

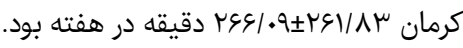

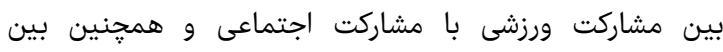

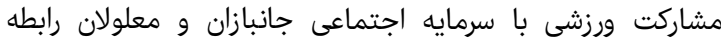

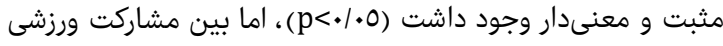




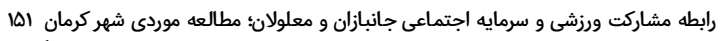

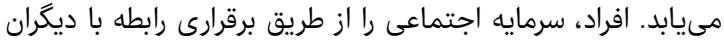

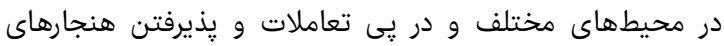

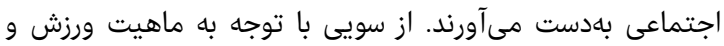

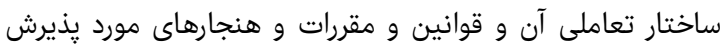

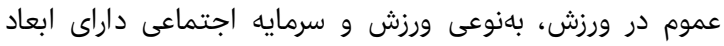

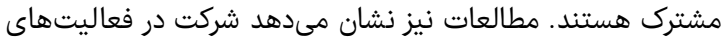

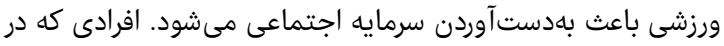

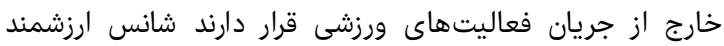

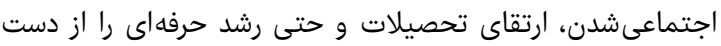

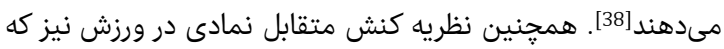

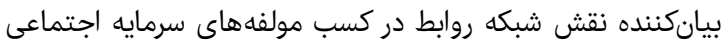

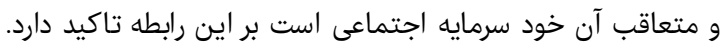

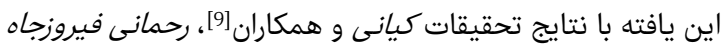

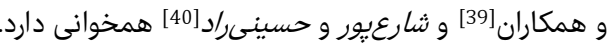

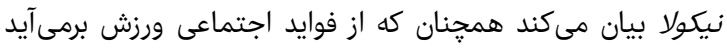

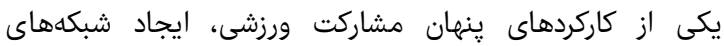

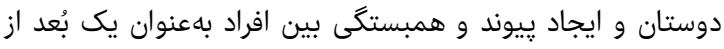

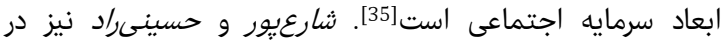

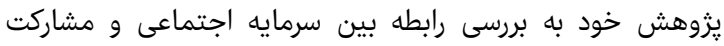

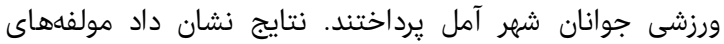

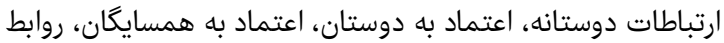

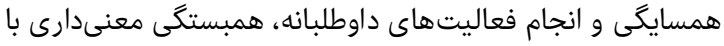

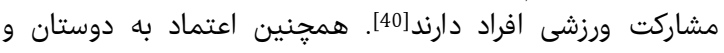

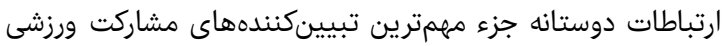

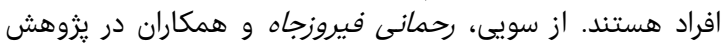

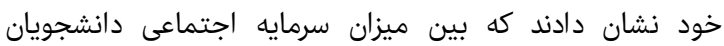

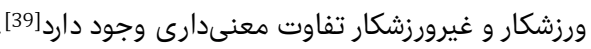

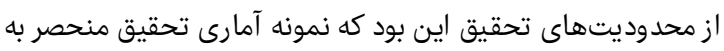

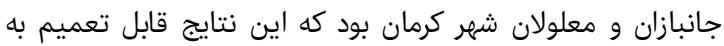

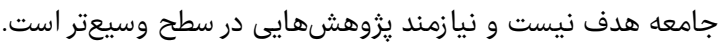

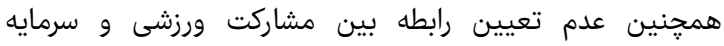

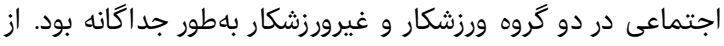

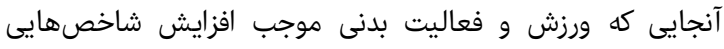

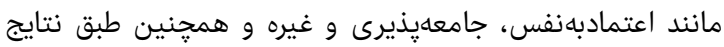

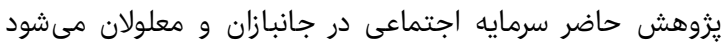

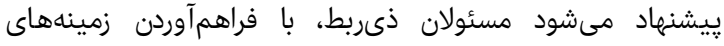

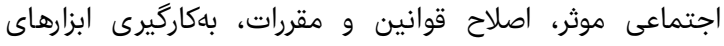

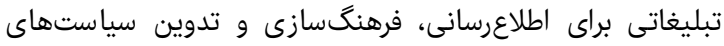

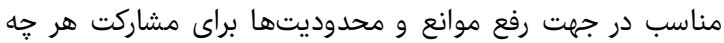

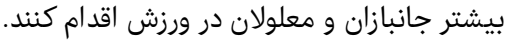

\section{نتيجه}

مشاركت ورزشى جانبازان و معلولان شهر كرمان با سرمايه اجتماعى

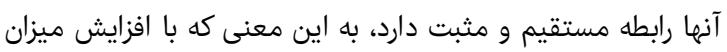

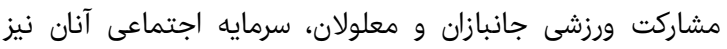
افزايش مىيابد.

تشكر و قدردانى: لازم است از همكارى صميمانه كليه جانبازان و

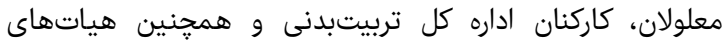

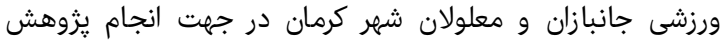
تقدير و تشكر نماييم.

Iranian Journal of War and Public Health
كاهش مشاركت اجتماعى مردان و زنان منجر مىشود. همجنين

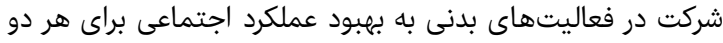
جنسيت (مرد و زن) مئ دانجامد.

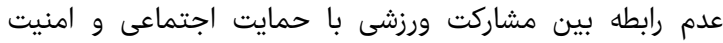

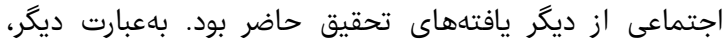

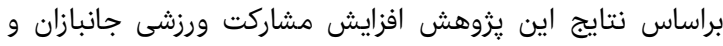

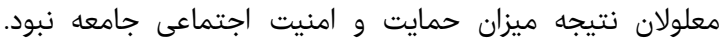

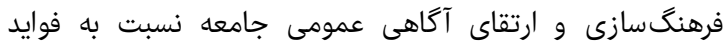

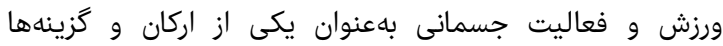

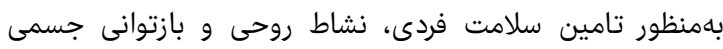

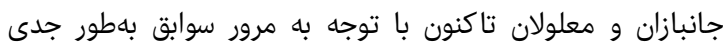

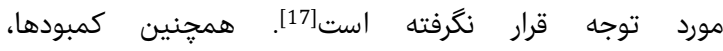

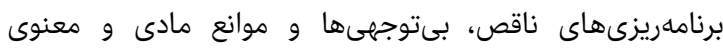

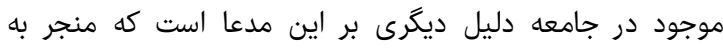

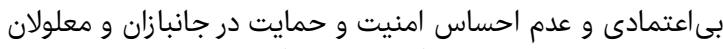

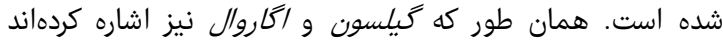

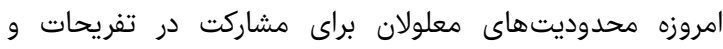

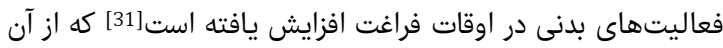

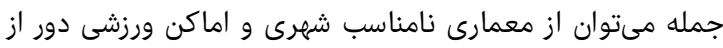

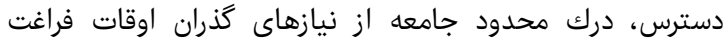

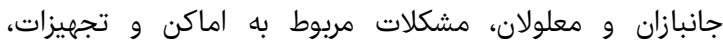

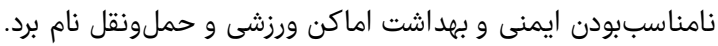

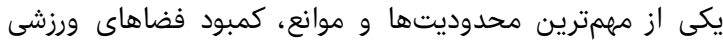

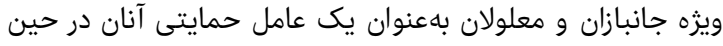

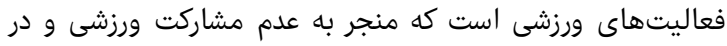

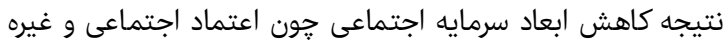

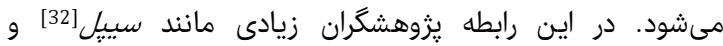

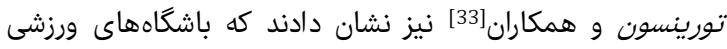

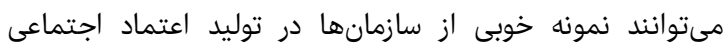

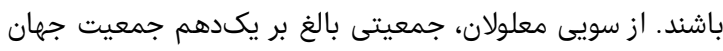

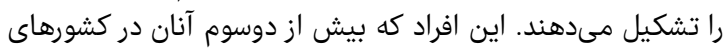

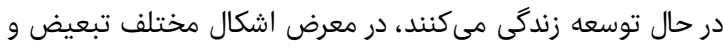

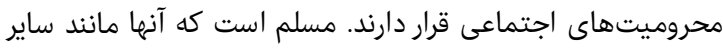

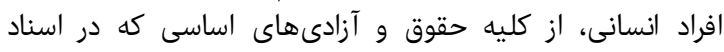

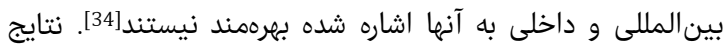

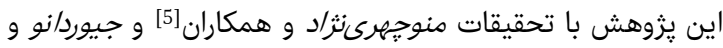

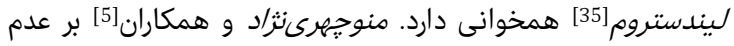

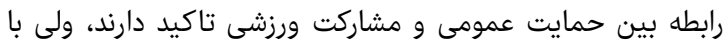

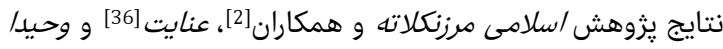

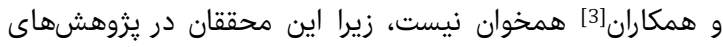

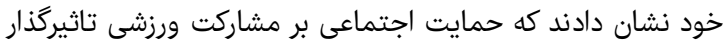

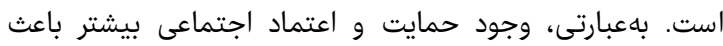

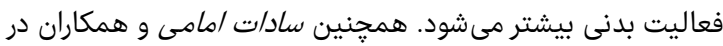

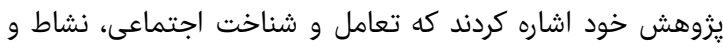

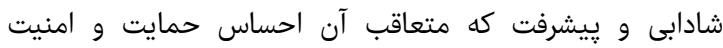

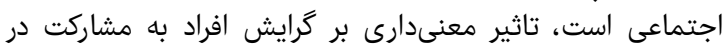

تفريحات ورزشى دارد[37]. نتايج يزوهش نشان داد بين مشاركت ورزشى و وردان سرمايه اجتماعى إنى

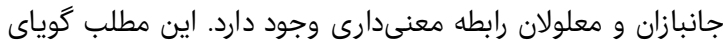

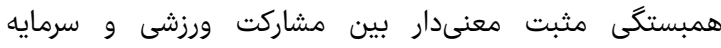

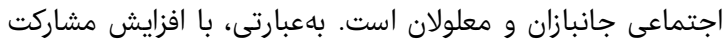

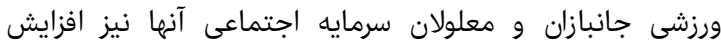


social capital and physical activity participation among adolescent. J Sport Manag. 2013;17(2):109-27. [Persian] 17- Razavi SM, Rajab Zadeh R, Mirzapour AA, Sajjadi SA. The role of physical activity and sport in leisure time of physical disabilities. J Sport Manag. 2013;9(18):125-41. [Persian]

18- Alizadeh MH, Gharakhanloo R. How to spend the leisure time of veterans and disabled (emphasizing the role of physical education and exercise). Harekat. 2000;6:23-40. [Persian]

19- White SM, Wójcicki T, McAuley E. Physical activity and quelity of life in community dwelling older adults. Health Qual Life Outcomes. 2009;7:10.

20-Bahmani F, Bakhshayesh H, Hamzehloui N. A study of the barriers to participation of the disabled, the blind, and the deaf in public sports in Arak city. Rese in spo manage. 2012;1(3):75-86. [Persian]

21- Rahmani A, Bakhshiniya T, Ghavami SS. The role of sport in leisure time spend of students in Islamic Azad University of Takestan. Res Sport Sci. 2006;4(3):33-50. [Persian]

22- Jaarsma EA, Dijkstra PU, Geertzen JH, Dekker R. Barriers to and facilitators of sports participation for people with physical disabilities: A systematic review. Scand J Med Sci Sports. 2014;24(6):871-81.

23- Ali Hosseini B, Bahrololom H, Hosseininia SR. The relationship between participation motives and sport commitment in disabled athletes. New Trends Sport Manag. 2014;2(6):43-54. [Persian]

24- Qodratnama A, Heydarinejad S. Relationship between sport participation motivation with rate of student's physical activity in Shahid Chamran University of Ahwaz. Sport Manag Rev. 2013;5(2):189-202. [Persian]

25- Laving JR. Sport commitment in wheelchair basketball: An interpretive look into the lives of individuals with physical disabilities [Dissertation]. Ontario, Canada: Brock University; 2012.

26- Ghasemi Pirbalooti A, Moemeni M, Khangah H. Compare body image of disabled athletes with disabilities and non-disabled male athlete. Arch Rehabi. 2009;10(4):26-31. [Persian]

27- Coackley JJ. Sport in society- issues and controversies. 7th edition. Boston: McGraw-Hill; 2001. pp. 9-86.

28- Parsamehr M, Torkan R. A Survey about the relationship between social capital and sport's activities in participation. Iran J Nurs Res. 2012;7(26):69-79. [Persian]

29- Lindström M, Moghaddassi M, Merlo J. Social capital and leisure time physical activity: A population based multilevel analysis in Sweden. J Epidemiol Community Health. 2003;57(1):23-8.

30- Wendel-Vos GC, Schuit AJ, Tijhuis MA, Kromhout D. Leisure time physical activity and health-related quality of life: cross-sectional and longitudinal associations. Qual Life Res. 2004;13(3):667-77.

31- Mama S, Diamond p, McCurdy sh, Evans A, Lee R. Individual, social and environmental correlates of physical activity in overweight and obese African American and Hispanic women: A structural equation model analysis. Prev Med Rep. 2015;2:57-64.

32- Seippel 0. Sport and social capital. Acta Sociol. 2006;49(2):169-83.

33- Thorlindsson T, Valdimarsdottir M, Hrafn Jonsson S. Community social structure, social capital and

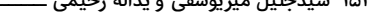

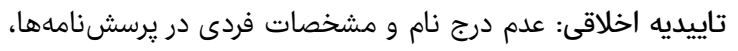

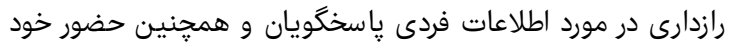

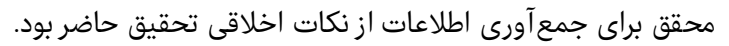
تعارض منافع: تعارض منافعى وجود نداشت أشات

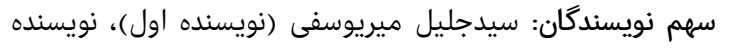

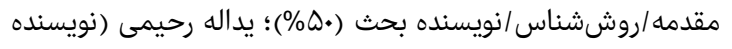

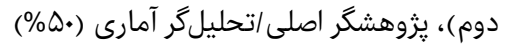

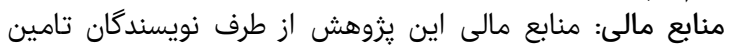

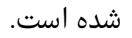
منابع

1- Safania AM. How to spend the leisure time of female students of Islamic Azad universities with an emphasis on sports activities. Harkat. 2001;9(9):127-40. [Persian] 2- Eslami Marzankolateh MM, Mousavi SJ, Khoshfar Gh. Relationship between social capital and participation in sports activities (case: Womens in Gorgan). Soc Capi Manag. 2014;1(2):139-159. [Persian]

3- Arizi F, Vahida F, Parsamehr M. Asurvey about impact of social support on the women participation sport activites. Olympic. 2006;14(1):77-86. [Persian]

4- Anvar Kholi A. Sport and society. Sheikh H, translator. Mashhad: Samt Press; 1994. p. 11. [Persian]

5- Manochehri Nezhad M, Hemmati Nezhad M, Rahmati MM. The relationship between family social capital and sport participation in adolescent students. J Sport Manag. 2015;7(3):457-70. [Persian]

6- Skinner J, Zakus D, Cowell J. Development through sport: Building social capital in disadvantaged communities. Sport Manag Rev. 2008;11(3):253-75.

7- Putnam RD. Bowling alone: The collapse and revival of American Community. $1^{\text {st }}$ edition. New York: Touchstone Books by Simon \& Schuster; 2001. pp. 1-10.

8- Coleman JS. Social capital in the creation of human capital. Am J Soc. 1988;94:S95-120.

9- Kiani M, Shirvani T, Ghanbari H, Kian S. Position on how to spend leisure time sports activities for the Veterans and Disabled. Iran J War Health. 2012;5(4):2634. [Persian]

10- Sajjadi H, Zanjari N. Disability in Iran: Prevalence, characteristics, and socio-economic correlates. Arch Rehabil. 2015;16(1):36-47. [Persian]

11- Majidi F, Teimouri S. A study of chaharbagh avenue as a case study to improve access for war-invalids and physical disabled people. Iran J War Health. 2011;3(11):36-44. [Persian]

12- Sobhani A, Andam R, Zarifi M. Study and prioritizing the factors and barriers to sport participation of Disabled people of Ahvaz city. Organ Behav Manag Sport Stud. 2015;2(6):41-8. [Persian]

13- Poursoltani $H$. The relationship between exercise and mental health of veterans and the disabled amputee in Iranian athletes. J Mov Sci Sports. 2003;1(2):10-7. [Persian]

14- Omar-Fauzee MS, Mohd-Ali M, Kim Geok S, Ibrahim N. The participation motive in the Paralympics. J Altern Perspect Soc Sci. 2010;2(1):250-72.

15- Powdthavee N. What happens to people before and after disability? Focusing effects, lead effects, and adaptation in different areas of life. Soc Sci Med. 2009;69(12):1834-44

16- Parsamehr M, Belgoorian M, Saeidi Madani M. The examination of the relationship between components of 


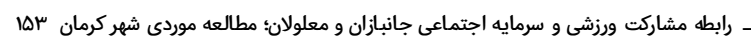
in University's Intramural Activities. Res Univ Sport. 2014;5(4):65-80. [Persian]

38- Allen JT, Daren DD, Byon KK, Mohn RS. Sport as a vehicle for socialization and maintenance of cultural identity: International students attending American universities. Sport manag Rev. 201013(4):421-34.

39- Rahmani FA, Sharepour M, Rezaei Pasha S. A study on the difference of social capital among the youthemphasizing on sport participation. Soc Stud Youth. 2011;2(2):37-62. [Persian]

40- Sharapour M, Hosseinirad A. Investigate the relationship between social capital and sport participation, (29-15-year-old citizen of the city of Babol). Harakat. 2008;37(1):133-53. [Persian] adolescent smoking: A multi-level analysis. Health Place. 2012;18(4):796-804.

34- Teh KC, Ong VT. Physical activity patterns of Singaporeans in 2001. Singapore Med J. 2004;45(11):517-29.

35- Giordano GN, Lindström M. Social capital and change in psychological health over time. Soc Sci Med. 2011;72(8):1219-27.

36- Enayat H, Movahed M, Heydary A. The study of the relationship between social trust and feeling of social security among youths in shiraz and Yasuj cities. J Appl Soc. 2012;23(1):81-104. [Persian]

37- Sadat Emami S, Heydarinejhad S, Shafinya P. Motivational Factor Analysis of Student Participation 